\title{
High Cardiorespiratory Fitness Is Associated with Reduced Risk of Low Bone Density in Postmenopausal Women
}

\author{
Laura F. DeFina, MD, David Leonard, PhD, Benjamin L. Willis, MD, Carolyn E. Barlow, PhD, \\ Carrie E. Finley, MS, Marjorie R. Jenkins, MD, Barbara C. Pence, PhD, Yan Zhang, PhD, \\ Ming-Chien Chyu, PhD, E. Michael Lewiecki, MD, and Chwan-Li Shen, PhD',3
}

\begin{abstract}
Purpose: The goal of this study was to determine the association between cardiorespiratory fitness (CRF) and bone mineral density (BMD) of the femoral neck (FN) in postmenopausal women using existing Cooper Center Longitudinal Study data.

Materials and Methods: A cohort of 1,720 predominantly healthy Caucasian women (57.1 \pm 6.9 years) underwent preventive medical examinations that included CRF assessment by maximal Balke treadmill testing and measurement of BMD by dual-energy X-ray absorptiometry. CRF was estimated from total treadmill time and categorized into five categories of $\mathrm{CRF}$ (further defined as fitness category $1=$ low fitness, $2-3=$ moderate fitness, and 4-5=high fitness). Logistic regression was used to characterize the association between CRF and $\mathrm{BMD}$, adjusting for age, weight, and resistance activity level.

Results: Overall, the mean body-mass index (BMI) for all subjects was $25.0 \pm 4.5 \mathrm{~kg} / \mathrm{m}^{2}$, although BMI was in the obese range in the low fitness group. The prevalence of osteoporosis (T-score $\leq-2.5$ at the FN) was greater in the low fit group than moderate or high fit $(5.8 \%$ vs. $3.0 \%$ or $3.9 \%$, respectively); with a similar pattern seen for prevalence of osteopenia (T-score $>-2.5$ and $\leq-1.0$ at the $\mathrm{FN})(47.5 \%$ vs. $46.4 \%$ or $44.8 \%$, respectively). Higher age and lower weight were associated with low BMD. Fully adjusted logistic regression models showed an inverse association between CRF and low BMD of the FN. For T-score $\leq-1.0$, the primary outcome, the odds ratio (OR) was 0.50 (95\% confidence interval [CI] 0.32-0.79) for moderate fitness, and OR of $0.32(95 \%$ CI $0.21-0.51)$ for high fitness was seen. For T-score $\leq-2.5$ at the FN, OR was 0.30 (95\% CI $0.11-0.80)$ for moderate fitness, and OR was 0.29 (95\% CI 0.12-0.71) for high fitness.
\end{abstract}

Conclusion: Increased CRF levels are associated with reduced risk for low bone density in postmenopausal women.

Keywords: fitness, osteoporosis, BMD, risk

\section{Introduction}

O STEOPOROSIS IS CHARACTERIZED by low bone mass and structural deterioration of bone tissues, leading to compromised bone strength and increased risk of fractures. ${ }^{1}$ In the United States, it is estimated that by 2020 , osteopenia (defined as a bone mineral density [BMD] T-score $>-2.5$ and $\leq-1.0$ ) or osteoporosis (T-score $\leq-2.5$ ) will affect more than 61 million women and men aged 50 years and older. ${ }^{2}$ The estimated cost of osteoporosis-related medical care is high and will rise to $\$ 25.3$ billion by $2025 .{ }^{3}$ Compared with men, women are four times more likely to develop osteoporosis due to a decline of estrogen levels after menopause. ${ }^{4}$ A rapid reduction in BMD usually occurs in the first 3-5 years immediately following menopause, with a slower rate of BMD reduction continuing throughout the remainder of a woman's life. ${ }^{1,4}$ For these reasons, the present study focused exclusively on women.

Regular weight-bearing and muscle-strengthening exercise is a universal recommendation for the prevention and treatment of osteoporosis, as well as to reduce the risk of falls and fractures in postmenopausal women and men

\footnotetext{
${ }^{1}$ The Cooper Institute, Dallas, Texas.

${ }^{2}$ Laura W. Bush Institute for Women's Health, Texas Tech University Health Sciences Center, Lubbock, Texas.

Departments of ${ }^{3}$ Pathology and ${ }^{4}$ Community and Family Medicine, Texas Tech University Health Sciences Center, Lubbock, Texas.

${ }^{5}$ Healthcare Engineering Program, Department of Mechanical Engineering, Texas Tech University, Lubbock, Texas.

${ }^{6}$ New Mexico Clinical Research \& Osteoporosis Center, Albuquerque, New Mexico.
} 
aged 50 years and older. ${ }^{5}$ Studies have shown that weightbearing exercise and strength training mitigate BMD loss in postmenopausal women. ${ }^{6,7}$ There is evidence that aerobic activity with moderate to high impact, such as jogging, will modestly increase BMD. However, there is limited evidence ${ }^{8,9}$ demonstrating that women or men with higher cardiorespiratory fitness (CRF) levels, which represent an objective marker of habitual physical activity, ${ }^{10}$ have lower risk for bone loss.

Based on the existing research, we hypothesized that lower levels of CRF in postmenopausal would be associated with increased risk of low bone density (T-score $\leq-1.0$ ) of the femoral neck $(\mathrm{FN})$. Given that the international reference standard for diagnosing osteoporosis is the FN, BMD at this skeletal site was selected as the primary endpoint of interest. Therefore, the goal of this cross-sectional study was to understand the relationship between objectively measured CRF and risk of low BMD (T-score $\leq-1.0$ ) in postmenopausal women who participated in the Cooper Center Longitudinal Study (CCLS).

\section{Materials and Methods}

\section{Study design}

This study is a retrospective cross-sectional analysis of an ongoing observational cohort, the CCLS, which was initiated in 1970 to evaluate the relationship between CRF and health outcomes. The CCLS cohort includes patients seen at the Cooper Clinic, a preventive medicine practice in Dallas, Texas. The data acquired at Cooper Clinic for the CCLS are not based on a systematic research protocol, but rather on the basis of clinical evaluation and recommendations from the patients' physicians. Thus, the availability of particular measurements may be limited due to physicians' clinical discretion and may vary from patient to patient. Participants signed an informed consent for the use of their data for research. The CCLS database is maintained by The Cooper Institute. Privacy precautions are maintained through The Cooper Institute policies. The data collection and informed consent are reviewed and approved annually by The Cooper Institute Institutional Review Board.

\section{Study population}

The current study population includes women who underwent both CRF and BMD testing at the same visit between 1998 and 2015. Participants are community-dwelling, generally healthy postmenopausal women who are either selfreferred or referred by their employers for preventive health examinations that include an extensive medical history questionnaire, a standardized medical examination by a physician, anthropometric measurements, fasting laboratory studies, a maximal treadmill exercise test, and screening BMD testing.

Beginning with a sample of 1,950 postmenopausal women who had both a treadmill test and a BMD test between 1998 and 2015, we excluded women who (a) had missing weight or resistance activity $(n=53)$, (b) were taking bisphosphonates $(n=105)$, or (c) had a history of cancer other than skin cancer (to exclude patients who received therapy that could impact BMD) $(n=72)$. This resulted in an analytic sample of 1,720 individuals.

\section{Measurement of health status}

At the index visit, study participants provided their medical history, which included self-reported medical, social, and physical activity history confirmed by the clinic physician. Menopausal status was defined as postmenopausal based on self-report or age $\geq 50$ years. Of note, the medical history did not contain questions about fragility fractures until 2010; hence, this variable was not included in the current study. Participants underwent a physical examination, including height and weight measurements using a standard clinical stadiometer and scale. Body-mass index (BMI) was calculated as weight in kilograms over height in meters squared. Laboratory measurements included alkaline phosphatase (ALP), creatinine, calcium, phosphorus, hemoglobin, thyroid-stimulating hormone (TSH), and 25 hydroxy-vitamin $\mathrm{D}[25(\mathrm{OH}) \mathrm{D}]$. The $25(\mathrm{OH}) \mathrm{D}(\mathrm{D} 2+\mathrm{D} 3)$ levels were measured on a DiaSorin Liaison Chemiluminescence Analyzer. The test-retest coefficient of variation for this assay was $\pm 11 \%$. The laboratory measurements, based on single analyses due to the clinical nature of population, were performed in accordance with standard procedures.

\section{Physical activity assessment}

Detailed physical activity, both aerobic and resistance, questions are part of the medical history questionnaire. Questions include information on the type, frequency, and duration of aerobic activity undertaken on a weekly basis over the preceding 3 months. The types of activities include walking, jogging or running, treadmill use, elliptical trainer, bicycling, stationary cycle, swimming, aerobic dance or floor exercise, vigorous activity (e.g., racquetball, singles tennis, skating), and other vigorous activity (e.g., basketball, soccer). Utilizing the Compendium of Physical Activities, these results are then converted into $\mathrm{MET} \cdot$ minutes of aerobic activity per week. ${ }^{11}$

With respect to resistance activities, the medical history contains information related to duration and frequency of the activity. The 2008 Physical Activity Guidelines for Americans recommend obtaining greater than or equal to 2 nonconsecutive days per week of resistance activities. ${ }^{12}$ Therefore, resistance activities were categorized into two groups: (a) greater than or equal to 2 days/week or (b) less than 2 days/week.

\section{CRF measurement}

For individuals without a medical or orthopedic contraindication, exercise testing is a standard component of the basic examination. $\mathrm{CRF}$ was estimated from the maximal time on a treadmill test using the modified Balke protocol; time on the treadmill with this protocol is highly correlated $(r=0.92)$ with measured $\mathrm{VO}_{2} \max$ (maximal oxygen consumption) in women. ${ }^{13}$ Maximal metabolic equivalent level $(1 \mathrm{MET}=$ $3.5 \mathrm{~mL} \mathrm{O}{ }_{2} \cdot \mathrm{kg}^{-1} \cdot \mathrm{min}^{-1}$ ) was estimated from the final treadmill speed and grade. ${ }^{14}$ Treadmill times were compared with age-specific normative data on treadmill performance within the entire CCLS so that treadmill time could be classified into age-specific categories of fitness (1-5, with 1 being the lowest level of fitness) for each participant. ${ }^{15} \mathrm{CRF}$ was then categorized as low fitness (category 1), moderate fitness (categories 2 and 3), and high fitness (categories 4 and 5). ${ }^{16}$ While no uniform consensus for the precise range of low 
fitness exists, category 1 has been considered low fitness in prior work ${ }^{16}$ and was associated with increased morbidity and mortality. ${ }^{15,17,18}$ These five categories are derived from five traditional health-related fitness categories from the CCLS as a whole and not on quintiles of the present sample.

\section{$B M D$ measurement}

Central BMD was measured by dual-energy X-ray absorptiometry (DXA) at the FN, lumbar spine, and total hip with a GE Lunar iDXA machine (GE Healthcare Lunar, Madison, WI) or Hologic Discovery QDR Series (Hologic, Bedford, MA) bone density units using quality control and calibration standards as specified by the manufacturer. Daily quality control checks are done utilizing a phantom. ${ }^{19}$ In this clinical population, body fat and lean mass assessments are not part of the routine BMD testing protocol and are generally done on less than $5 \%$ of this population. Thus, these variables were not included in this analysis.

The primary outcome of interest was the lowest $\mathrm{T}$-score of the left and right FN. This was selected because FN T-score is the World Health Organization reference standard for the diagnosis of osteoporosis. BMD was also assessed in total hip; L1-L4; and lowest of the cumulative T-scores of FN, total hip, and/or L1-L4 for secondary outcomes. For purposes of defining prevalence in this population, osteoporosis was defined as T-score $\leq-2.5$, and osteopenia was defined as T-score $>-2.5$ and $\leq-1.0$, using the World Health Organization definitions and reference population. ${ }^{20}$ For regression modeling, thresholds of T-score $\leq-2.5$ (osteoporosis) and $\mathrm{T}$-score $\leq-1.0$ (osteoporosis and osteopenia) were utilized.

\section{Statistical analyses}

Descriptive characteristics of the study sample were calculated as means and standard deviations for continuous variables and percentages for categorical variables. We used the Jonckheere-Terpstra nonparametric method to test for trends of characteristics across ordered fitness levels. We fit multiple logistic regression models of T-score $\leq-1.0$ to estimate adjusted odds ratios (ORs) for a set of prespecified covariates: age, weight, CRF level, and resistance activity level. Secondary analyses included T-score $\leq-2.5$ as an outcome; vitamin D was included as a covariate in an analysis of the subgroup with vitamin D available; and current smoking was included as a covariate in a subgroup analysis of those with definitive smoking status. Tests of Spearman's correlation and partial correlation coefficients were based on Fisher's $z$-transformation. All analyses were programmed in SAS/STAT ${ }^{\circledR}$ statistical software, version 9.4.

\section{Results}

In the study population of 1,720 women, the average age was $57.1 \pm 6.9$ years. The majority of the sample self-selected as non-Hispanic white $(n=1,576,91.6 \%$ of the sample). Table 1 presents the anthropometric and physiological measurements according to CRF categories. The mean BMI was $25.0 \pm 4.5 \mathrm{~kg} / \mathrm{m}^{2}$. In the moderate fitness group (categories 2 and 3), the average BMI was in the overweight (BMI 25$29.9 \mathrm{~kg} / \mathrm{m}^{2}$ ) range, while in the low fitness group (category 1 ), it was in the obese $\left(\right.$ BMI $\left.\geq 30 \mathrm{~kg} / \mathrm{m}^{2}\right)$ range $(p<0.001)$. Aerobic activity and resistance activity positively corresponded to the levels of CRF categories (both $p<0.001$ ).
Laboratory data showed normal serum ALP, renal function (serum creatinine), calcium and phosphorus, hematocrit, and thyroid function (TSH) (Table 1). There was no significant difference in serum calcium ( $p=0.93$ for trend), phosphorus ( $p=0.23$ for trend), and TSH concentrations $(p=0.86$ for trend) among different CRF groups. ALP concentration decreased from low to high fitness groups; while hemoglobin and $25(\mathrm{OH}) \mathrm{D}$ levels increased from low to high fitness groups (all $p<0.006$ ). Both moderate and high fitness groups had normal mean serum 25(OH)D levels $(\geq 30 \mathrm{ng} / \mathrm{mL})$. However, those in the low fitness group had a lower mean serum $25(\mathrm{OH}) \mathrm{D}$ level $(28.5 \pm 18.3 \mathrm{ng} / \mathrm{mL})$. Table 2 shows the relationship between BMD status and categorical fitness. The highest prevalence of osteoporosis and osteopenia was found in the low fitness group.

Multivariable adjusted ORs for the presence of T-score $\leq$ -1.0 in the FN, the primary analysis, and $\leq-2.5$ in the FN are presented in Tables 3 and 4. Older age and lower body weight were associated with greater risk of low BMD of the FN. Relative to low fitness, moderate and high fitness were associated with lower odds of low BMD at the FN by either Tscore cut point $(\mathrm{T}$-score $\leq-1.0-\mathrm{OR} 0.50,95 \%$ confidence interval [CI] 0.32-0.79; OR 0.32, 95\% CI $0.21-0.51$ and Tscore $\leq-2.5-$ OR $0.30,95 \%$ CI $0.11-0.80$; OR $0.29,95 \%$ CI $0.12-0.71)$. Self-reported resistance activity was not associated with T-score $\leq-1.0$ or $\leq-2.5$ at the FN. In the subgroup of 1,575 women with definitive smoking status (data not shown), current smoking was not associated with T-score $\leq$ -1.0 at the FN (OR $1.49,95 \%$ CI $0.86-2.58$ ) or with T-score $\leq-2.5$ at the FN (OR 2.41, 95\% CI 0.87-6.66). Supplementary Tables S1-S3 (Supplementary Data are available online at www.liebertpub.com/jwh) show the additional relationships between fitness and BMD of the total hip, lumbar spine (L1-L4), and a cumulative outcome involving the lowest T-score of the FN, total hip, and L1-L4.

Table 5 shows the adjusted OR for the T-score $\leq-1.0$ or $\leq$ -2.5 in the subgroup of 898 women with concurrent vitamin D measurements. Relative to low fitness, moderate and high fitness were associated with lower odds of FN-BMD T-score $\leq-1.0$ (OR 0.48, 95\% CI 0.26-0.90; OR 0.32, 95\% CI 0.17-0.59). High fitness relative to low fitness was associated with lower odds of FN T-score $\leq-2.5$ (OR $0.27,95 \%$ CI $0.08-0.95)$, but moderate fitness was not (OR $0.29,95 \%$ CI $0.08-1.09$ ). No association was observed between $25(\mathrm{OH}) \mathrm{D}$ and lower BMD when adjusting for all covariates $(p=0.738$ and $p=0.113)$.

Further evaluation of the relationship between BMD and resistance activity or fitness was undertaken using Spearman's correlation coefficients. The Spearman partial correlation coefficient relating FN BMD to resistance activities (minutes/week) was $0.08(p=0.001)$, adjusted for weight. The Spearman partial correlation coefficient relating FNBMD to treadmill time was $0.22(p<0.001)$, adjusted for weight. These results suggest a stronger relationship between fitness and BMD than between resistance activity and BMD.

\section{Discussion}

This cross-sectional analysis of a large cohort of generally healthy postmenopausal women demonstrated that those with higher levels of CRF had lower odds of T-score $\leq-1.0$ and $\leq$ -2.5 at the FN than those whose fitness was low. The reduced risk of osteopenia and osteoporosis was comparable in the 
Table 1. Baseline Characteristics of Postmenopausal Women with Bone Mineral Density Testing in the Cooper Center longitudinal Study by Standard Fitness Categories

\begin{tabular}{lccccc}
\hline & Low fit ${ }^{\mathrm{a}}, \mathrm{N}=120$ & Moderate $_{\text {fit }}^{\mathrm{a}}, \mathrm{N}=461$ & High fit $^{\mathrm{a}}, \mathrm{N}=1,139$ & All, $\mathrm{N}=1,720$ & $\mathrm{p}$ \\
\hline Age (years) & $57.1 \pm 7.9$ & $57.5 \pm 7.6$ & $56.9 \pm 6.4$ & $57.1 \pm 6.9$ & 0.10 \\
Weight (kg) & $82.1 \pm 16.5$ & $72.5 \pm 13.5$ & $64.1 \pm 10.1$ & $67.6 \pm 12.8$ & $<0.001$ \\
Body-mass index (kg/m ${ }^{2}$ ) & $30.8 \pm 5.9$ & $26.9 \pm 4.7$ & $23.6 \pm 3.4$ & $25.0 \pm 4.5$ & $<0.001$ \\
Current drinker, yes (\%) & $68(56.7)$ & $328(71.1)$ & $867(76.1)$ & $1263(73.4)$ & $<0.001$ \\
Current smoker, yes (\%) & $9(7.5)$ & $24(5.2)$ & $29(2.5)$ & $62(3.6)$ & $<0.001$ \\
Laboratory values & & & & & \\
$\quad$ Alkaline phosphatase (U/L) & $88.5 \pm 29.5$ & $77.5 \pm 23.0$ & $69.1 \pm 20.8$ & $72.9 \pm 22.9$ & $<0.001$ \\
Creatinine (mg/dL) & $0.83 \pm 0.19$ & $0.81 \pm 0.16$ & $0.80 \pm 0.16$ & $0.80 \pm 0.16$ & 0.15 \\
Calcium (mg/dL) & $9.3 \pm 0.4$ & $9.3 \pm 0.4$ & $9.3 \pm 0.4$ & $9.3 \pm 0.4$ & 0.93 \\
Phosphorus (mg/dL) & $3.7 \pm 0.5$ & $3.7 \pm 0.5$ & $3.7 \pm 0.5$ & $3.7 \pm 0.5$ & 0.23 \\
Hemoglobin (g/dL) & $13.4 \pm 1.3$ & $13.6 \pm 0.9$ & $13.7 \pm 0.9$ & $13.6 \pm 0.9$ & 0.006 \\
TSH (IU/L) & $2.5 \pm 3.2$ & $2.5 \pm 4.1$ & $2.3 \pm 2.1$ & $2.4 \pm 2.9$ & 0.86 \\
Serum 25(OH)D (ng/mL) & $28.5 \pm 18.3$ & $30.0 \pm 12.6$ & $34.7 \pm 14.4$ & $32.9 \pm 14.5$ & $<0.001$ \\
Activity parameters & & & & & \\
Physical activity (MET·minutes/ & $371.0 \pm 780.8$ & $612.3 \pm 879.8$ & $1185.5 \pm 1357.2$ & $975.0 \pm 1248.6<0.001$ \\
$\quad$ week) & & & & & \\
Resistance activity (days/week) & $0.5 \pm 1.1$ & $0.6 \pm 1.3$ & $1.4 \pm 1.6$ & $1.1 \pm 1.5$ & $<0.001$ \\
Resistance activity (minutes/week) & $16.6 \pm 41.9$ & $20.2 \pm 45.9$ & $52.2 \pm 76.6$ & $41.2 \pm 69.4$ & $<0.001$ \\
Cardiorespiratory fitness (MET) & $5.8 \pm 0.8$ & $7.4 \pm 0.7$ & $9.7 \pm 1.4$ & $8.8 \pm 1.8$ & $<0.001$ \\
\hline
\end{tabular}

Data presented as mean $\pm \mathrm{SD}$ or $N(\%)$ when categorical.

${ }^{a}$ Low fit, category 1 age-specific normative data within the CCLS; moderate fit, categories 2-3; high fit, categories 4-5. $\mathrm{b}_{n=898 \text {. }}$

CCLS, Cooper Center Longitudinal Study; 25(OH)D, 25 hydroxy-vitamin D; TSH, thyroid-stimulating hormone.

moderate and high fitness groups versus the low fitness group, suggesting that even modest differences in CRF could be associated with skeletal benefit. These findings align well with the concept that regular exercise is beneficial for bone health. To our knowledge, this is the largest study to show that higher CRF, a marker of habitual physical activity, is associated with lower risk of osteoporosis and osteopenia involving critical skeletal areas in otherwise healthy postmenopausal women.

The types of physical activity necessary for optimal bone health likely differ from those necessary for optimal cardiovascular health. Whereas the intensity of activity required to raise heart rate is a critical factor for cardiovascular health, the peak load or impact is likely more important for bone health. $^{21,22}$ Thus, weight-bearing activities have been considered the primary exercise for bone health. Resistance training can increase muscle size and strength, ${ }^{23,24}$ potentially leading to higher BMD at the muscle site. ${ }^{25,26}$ There is evidence, however, supporting the benefit of aerobic activity on bone health. In a 12-year follow-up of the Nurses' Health
Study ( $n=61,200$ postmenopausal women, aged 40-77 years), authors reported that moderate levels of aerobic activity, including walking, were associated with substantially lower risk of hip fracture in postmenopausal women. ${ }^{27} \mathrm{Ac}-$ tive women with at least $24 \mathrm{MET} \cdot$ hours per week had a $55 \%$ lower risk of hip fracture compared with sedentary women with less than $3 \mathrm{MET}$-hours per week. ${ }^{27}$ However, in that study, no BMD data were available to evaluate the association between aerobic activity level and BMD. An association of higher levels of physical activity with lower hip fracture risk has been documented in several observational studies. $^{28,29}$ Plausible mechanisms include maintenance of muscle mass and BMD, improved balance, decreased risk of falls, and enhanced vitamin D status. ${ }^{30,31}$

Our findings that low objectively measured CRF was associated with increased risk of low BMD extends work done by others to the important population of postmenopausal women. ${ }^{8,9,29,32,33}$ For example, in a group of young Lebanese adults (18-32 years, 20 women and 37 men), El Hage et al. ${ }^{9}$ reported that $\mathrm{VO}_{2}$ max is a positive determinant of bone mineral content

Table 2. Femoral Neck Bone Mineral Density Status by Fitness Categories

\begin{tabular}{lccc}
\hline Femoral neck & Low fit ${ }^{\mathrm{a}}, \mathrm{N}=120$ & Moderate fit ${ }^{\mathrm{a}}, \mathrm{N}=461$ & High $^{\mathrm{a}} \mathrm{i}^{\mathrm{a}}, \mathrm{N}=1,139$ \\
\hline Bone mineral density $\left(\mathrm{g} / \mathrm{cm}^{2}\right)$ & $0.88 \pm 0.13$ & $0.89 \pm 0.12$ & $0.89 \pm 0.12$ \\
T-score & $-1.02 \pm 1.00$ & $-0.88 \pm 0.96$ & $-0.90 \pm 0.96$ \\
Osteoporosis $^{\mathrm{b}}$ & $7(5.8)$ & $14(3.0)$ & $44(3.9)$ \\
Osteopenia $^{\mathrm{c}}$ & $57(47.5)$ & $214(46.4)$ & $510(44.8)$ \\
Normal $^{2}$ & $56(46.7)$ & $233(50.5)$ & $585(51.4)$ \\
\hline
\end{tabular}

Data presented as mean $\pm \mathrm{SD}$ or $N(\%)$ when categorical.

${ }^{a}$ Low fit, category 1 based on age-specific normative data within the CCLS; moderate fit, categories 2 and 3; high fit, categories 4 and 5.

${ }^{\mathrm{b}} \mathrm{T}$-score $\leq-2.5$.

c $-2.5<$ T-score $\leq-1.0$. 

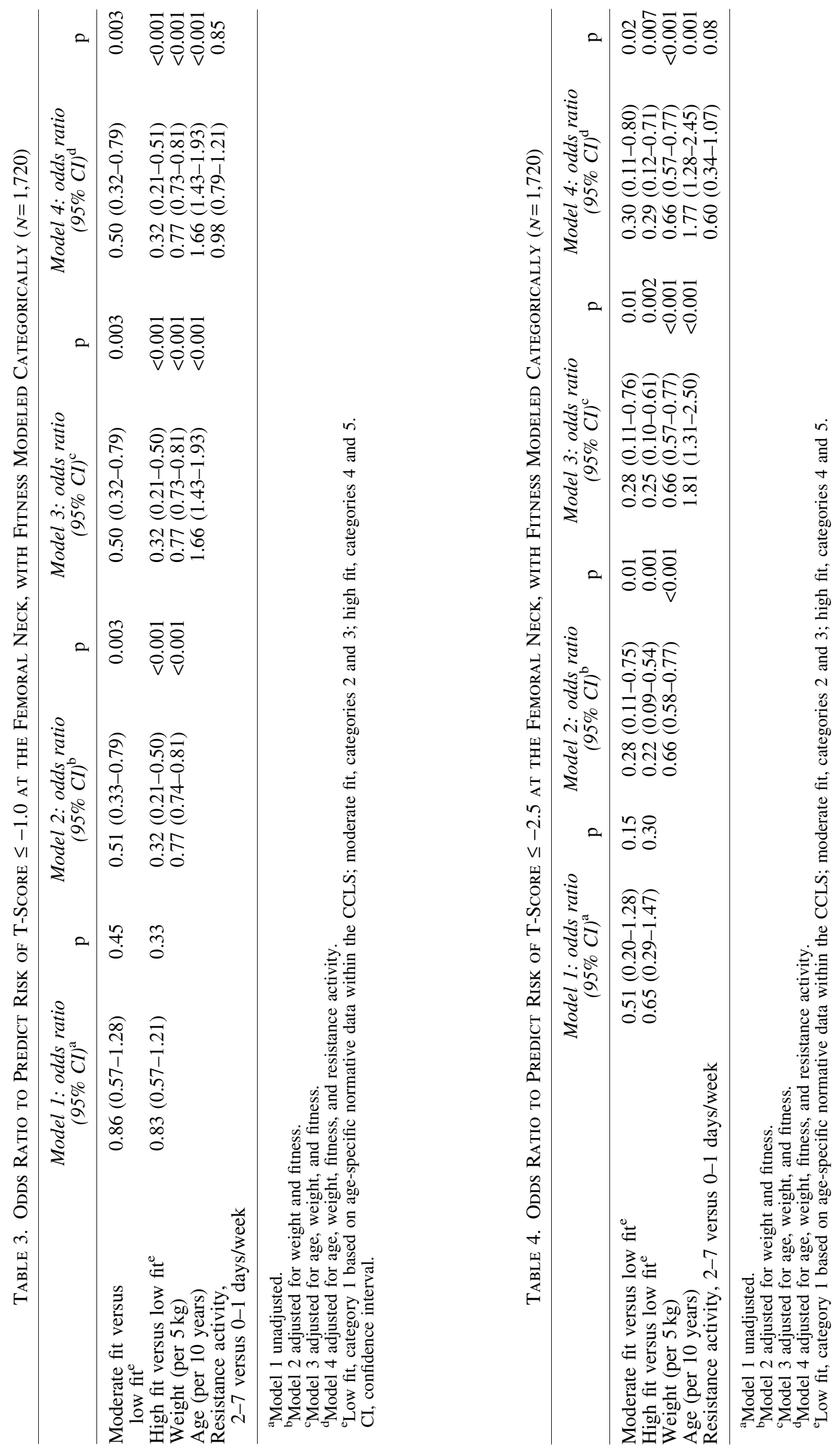
Table 5. Odds Ratio to Predict Risk of T-Score $\leq-1.0$ and $\leq-2.5$ at the Femoral Neck, with Fitness Modeled Categorically, Vitamin D Subgroup $(N=898)$

\begin{tabular}{|c|c|c|c|c|}
\hline & \multicolumn{4}{|c|}{$T$-score at femoral neck $\leq-2.5$} \\
\hline & \multicolumn{2}{|c|}{$T$-score at femoral neck $\leq-1.0$} & \multicolumn{2}{|c|}{$T$-score at femoral neck $\leq-2.5$} \\
\hline & Odds ratio $(95 \% C I)^{\mathrm{a}}$ & $\mathrm{p}$ & Odds ratio $(95 \% C I)^{\mathrm{a}}$ & $\mathrm{p}$ \\
\hline Age (per 10 years) & $1.83(1.47-2.29)$ & $<0.001$ & $1.49(0.95-2.35)$ & 0.085 \\
\hline Weight (per $5 \mathrm{~kg}$ ) & $0.75(0.71-0.80)$ & $<0.001$ & $0.67(0.55-0.81)$ & $<0.001$ \\
\hline Moderate fit versus low fit ${ }^{b}$ & $0.48(0.26-0.90)$ & 0.022 & $0.29(0.08-1.09)$ & 0.067 \\
\hline High fit versus low fit ${ }^{\mathrm{b}}$ & $0.32(0.17-0.59)$ & $<0.001$ & $0.27(0.08-0.95)$ & 0.041 \\
\hline 25-Hydroxyvitamin D (per $5 \mathrm{ng} / \mathrm{mL}$ ) & $0.96(0.91-1.01)$ & 0.113 & $1.02(0.91-1.14)$ & 0.738 \\
\hline Resistance activity, $2-7$ versus $0-1$ days/week & $1.14(0.85-1.55)$ & 0.385 & $0.66(0.31-1.40)$ & 0.278 \\
\hline
\end{tabular}

${ }^{a}$ Model adjusted for age, weight, fitness, vitamin D, and resistance activity.

${ }^{b}$ Low fit, category 1 based on age-specific normative data within the CCLS; moderate fit, categories 2 and 3; high fit, categories 4 and 5.

and BMD in young adults. Schwarz et al. ${ }^{8}$ also reported that CRF was associated with BMD in 153 men aged 31-60 years.

The primary outcome evaluated in this study was the BMD of the FN. The secondary outcomes, including total hip, L1L4, and the cumulative measure of FN, total hip, and L1-L4, did not show a consistent relationship with fitness as seen in the Supplementary Tables S1-S3. In this large clinical cohort, we did not have the ability to visually assess whether the measured BMD was affected by concurrent degenerative changes. Osteoarthritis of the spine is known to be a major reason for hip-spine discordance in BMD. ${ }^{34}$ Furthermore, the active nature of this cohort may compound the likelihood of BMD elevation due to osteoarthritis as regular axial loadbearing physical activity is known to increase the risk of degenerative changes. Thus, the lack of an evident relationship between fitness and BMD of these regions requires further evaluation in a population where the additive effect of degenerative changes on BMD can be assessed.

As observed in this and other studies, advancing age is also associated with an increased risk of osteopenia and osteoporosis, as well as fracture, ${ }^{35}$ likely due to loss of bone matrix, degradation of bone structure, and changes in the material properties of bone. On the other hand, higher body weight was associated with a reduced risk of low BMD, possibly due to factors that include skeletal loading and hormonal effects of adipose tissue. The moderate and high fitness groups were more active and less likely to smoke, but they were also more likely to drink alcoholic beverages and have lower body weight. Thus, the impact of CRF on bone density is likely not strictly through effects on known risk factors for bone loss.

A conundrum in interpreting these results is the absence of statistically significant benefit of resistance activities when controlling for CRF levels. Possible explanations include (a) resistance activities are self-reported and limited in precision, while CRF is objectively measured; (b) those with higher CRF levels are also more likely to do some form of weight training activity and thus the benefit is not evident in this epidemiological assessment; and (c) in those doing adequate aerobic activity, with axial weight bearing, resistance activities may not be as critical in the maintenance of bone density.

Small, but statistically significant, differences in some laboratory parameters were observed. The clinical relevance of these differences is uncertain, but may reflect lifestyle differences beyond exercise behavior. For example, the slightly lower serum ALP levels in the higher fit groups could be a reflection of improved bone mineralization due to higher vitamin D levels. In this study, the lower risk of osteopenia and osteoporosis in the moderate and high fitness groups may be, in part, due to a higher serum $25(\mathrm{OH}) \mathrm{D}$ level. The slightly higher serum $25(\mathrm{OH}) \mathrm{D}$ in the fitter groups could be due to differences in diet, vitamin D supplementation, or sun exposure associated with outdoor activities. The findings of this inverse relationship between serum $25(\mathrm{OH}) \mathrm{D}$ levels and CRF levels were consistent with another study ${ }^{9}$ evaluating a different subcohort in the CCLS. Serum vitamin D insufficiency has been documented to coexist with low BMD in postmenopausal women. ${ }^{36,37}$ Still, in the subgroup of women with concurrent serum $25(\mathrm{OH}) \mathrm{D}$ measurement $(n=898)$, the $25(\mathrm{OH}) \mathrm{D}$ level did not predict osteoporosis or osteopenia of the FN when adjusted for age, weight, CRF, and resistance activity. Lack of correlation between serum 25(OH)D and prediction of osteoporosis or osteopenia may be due to a ceiling effect with a relative small range of $25(\mathrm{OH}) \mathrm{D}(25.5-$ $34.4 \mathrm{ng} / \mathrm{mL}$ ) in our study population.

The major strength of the present study is the large and well-characterized cohort of apparently healthy women who had objective measures of CRF, serum laboratory analyses, and BMD. We attempted to limit the potential confounding effects by adjusting for age, weight, resistance activity level, smoking status, and vitamin D level in our multivariate analyses. However, confounding factors with potential effects on BMD, such as estrogen therapy and dietary calcium intake, were not assessed due to limitations of the database. Interpretation of this analysis is limited by its cross-sectional study design and the small number of women with osteoporosis. Causality between CRF and BMD cannot be established, and time-dependent changes in any factor cannot be ascertained from this cross-sectional study. Although more exercise may improve both fitness and $\mathrm{BMD}$, it is also plausible that healthier women with better bones are more likely to exercise and be fit. Furthermore, the types of aerobic activity were diverse, and participants generally did not participate in isolated types of activities based on the history questionnaire; hence, an assessment of the best form of activity leading to CRF for enhanced BMD cannot be determined. Furthermore, the population is very homogeneous with greater than $90 \%$ being non-Hispanic white. While this potentially decreases the ability to generalize these findings, non-Hispanic white females are certainly at greater risk for 
osteoporosis than other racial and ethnic groups, suggesting that the benefits would be more evident in this population. Finally, while the relationship of CRF and BMD is promising, this study does not have long-term outcome data on fragility fractures as this variable has only been a part of the medical history questionnaire since 2010 .

The 2008 Physical Activity Guidelines for Americans recommend at least 150 minutes (or $500 \mathrm{MET} \cdot$ minutes/ week) of moderate intensity aerobic activity every week and muscle-strengthening activities on 2 or more days a week for adults for health benefits. ${ }^{12}$ Furthermore, based on the recommendation of the Surgeon General, the optimal goal for bone health is at least 30 minutes of physical activity on most days, preferably daily. ${ }^{37}$ As exercise is also associated with a risk of injury or even death, the expected benefits of exercise must be balanced with such potential risks. In a study evaluating 909 community-dwelling women, an increased risk of musculoskeletal injuries was seen with increasing activity, ${ }^{38}$ but the majority of injuries were minor in terms of severity and healthcare costs. ${ }^{39}$ Thus, for the vast majority of individuals, it is likely that the benefits (e.g., reduced risk factors for cardiovascular disease, lower body weight, and fewer falls, as well as higher BMD) will outweigh the risks.

\section{Conclusion}

In a large and well-characterized observational study of generally healthy postmenopausal women, we report a positive association between CRF and BMD. Our findings suggest that having even a moderate fitness level through regular physical activity should be a standard recommendation for prevention of bone loss in postmenopausal women. Practicing clinicians should utilize this information on the osteoprotective effects of CRF to more strongly encourage their female patients to exercise regularly. Further randomized controlled studies are needed to establish causality between CRF and skeletal health, define the dose-response relationship, and assess the impact of higher fitness on fragility fractures, as well as assessing the relationship in men.

\section{Acknowledgments}

The authors thank Kenneth H. Cooper, MD, MPH, for establishing the CCLS; the Cooper Clinic for collecting the data; and The Cooper Institute for data management. This study was supported by funds from the Texas Tech University Health Sciences Center, Lubbock, TX, to C.L.S.

\section{Author Disclosure Statement}

No competing financial interests exist.

\section{References}

1. NIH Consensus Development Panel on Osteoporosis Prevention, Diagnosis, and Therapy. Osteoporosis prevention, diagnosis, and therapy. JAMA 2001;285:785-795.

2. Boonen S, Dejaeger E, Vanderschueren D, et al. Osteoporosis and osteoporotic fracture occurrence and prevention in the elderly: A geriatric perspective. Best Pract Res Clin Endocrinol Metab 2008;22:765-785.

3. Burge R, Dawson-Hughes B, Solomon DH, Wong JB, King A, Tosteson A. Incidence and economic burden of osteoporosisrelated fractures in the United States, 2005-2025. J Bone Miner Res 2007;22:465-475.
4. Looker AC, Johnston CC, Jr., Wahner HW, et al. Prevalence of low femoral bone density in older U.S. women from NHANES III. J Bone Miner Res 1995;10:796-802.

5. National Osteoporosis Foundation. Clinician's guide to prevention and treatment of osteoporosis. Washington, DC: National Osteoporosis Foundation, 2013.

6. Gomez-Cabello A, Ara I, Gonzalez-Aguero A, Casajus JA, Vicente-Rodriguez G. Effects of training on bone mass in older adults: A systematic review. Sports Med 2012;42:301-325.

7. Howe TE, Shea B, Dawson LJ, et al. Exercise for preventing and treating osteoporosis in postmenopausal women. Cochrane Database Syst Rev 2011;7;CD000333.

8. Schwarz P, Jorgensen N, Nielsen B, Laursen AS, Linneberg A, Aadahl M. Muscle strength, power and cardiorespiratory fitness are associated with bone mineral density in men aged 31-60 years. Scand J Public Health 2014;42:773-779.

9. El Hage R, Zakhem E, Theunynck D, et al. Maximal oxygen consumption and bone mineral density in a group of young Lebanese adults. J Clin Densitom 2014;17:320-324.

10. Haskell WL, Lee IM, Pate RR, et al. Physical activity and public health: Updated recommendation for adults from the American College of Sports Medicine and the American Heart Association. Med Sci Sports Exerc 2007;39:1423-1434.

11. Ainsworth BE, Haskell WL, Whitt MC, et al. Compendium of physical activities: An update of activity codes and MET intensities. Med Sci Sports Exerc 2000;32:S498-S504.

12. Physical Activity Guidelines Advisory Committe. Physical activity guidelines advisory committee report, 2008. Washington, DC: Department of Health and Human Services, 2008.

13. Pollock ML, Foster C, Schmidt D, Hellman C, Linnerud AC, Ward A. Comparative analysis of physiologic responses to three different maximal graded exercise test protocols in healthy women. Am Heart J 1982;103:363-373.

14. American College of Sports Medicine. ACSM's guidelines for exercise testing and prescription, 6th ed. Philadelphia, PA: Lippincott Williams \& Wilkins, 2000.

15. Blair SN, Kohl HW, III, Paffenbarger RS, Jr., Clark DG, Cooper KH, Gibbons LW. Physical fitness and all-cause mortality: A prospective study of healthy men and women. JAMA 1989;262:2395-2401.

16. Willis BL, Morrow JR, Jr., Jackson AW, Defina LF, Cooper KH. Secular change in cardiorespiratory fitness of men: Cooper Center Longitudinal Study. Med Sci Sports Exerc 2011;43:2134-2139.

17. Kampert JB, Blair SN, Barlow CE, Kohl HW, III. Physical activity, physical fitness, and all-cause and cancer mortality: A prospective study of men and women. Ann Epidemiol 1996;6:452-457.

18. Sui X, Hooker SP, Lee IM, et al. A prospective study of cardiorespiratory fitness and risk of type 2 diabetes in women. Diabetes Care 2008;31:550-555.

19. Hind K, Oldroyd B, Truscott JG. In vivo precision of the GE Lunar iDXA densitometer for the measurement of totalbody, lumbar spine, and femoral bone mineral density in adults. J Clin Densitom 2010;13:413-417.

20. Kanis JA; on behalf of the World Health Organization Scientific Group. Assessment of osteoporosis at the primary health-care level. University of Sheffield, United Kingdom: World Health Organization Collaborating Centre for Metabolic Bone Diseases, 2007.

21. Kerr D, Morton A, Dick I, Prince R. Exercise effects on bone mass in postmenopausal women are site-specific and load-dependent. J Bone Miner Res 1996;11:218-225. 
22. Lanyon LE. Using functional loading to influence bone mass and architecture: Objectives, mechanisms, and relationship with estrogen of the mechanically adaptive process in bone. Bone 1996;18:37S-43S.

23. Fiatarone MA, Marks EC, Ryan ND, Meredith CN, Lipsitz LA, Evans WJ. High-intensity strength training in nonagenarians. Effects on skeletal muscle. JAMA 1990;263: 3029-3034.

24. Taaffe DR, Duret C, Wheeler S, Marcus R. Once-weekly resistance exercise improves muscle strength and neuromuscular performance in older adults. J Am Geriatr Soc 1999;47:1208-1214.

25. Kelley GA. Exercise and regional bone mineral density in postmenopausal women: A meta-analytic review of randomized trials. Am J Phys Med Rehabil 1998;77:76-87.

26. Layne JE, Nelson ME. The effects of progressive resistance training on bone density: A review. Med Sci Sports Exerc 1999;31:25-30.

27. Feskanich D, Willett W, Colditz G. Walking and leisuretime activity and risk of hip fracture in postmenopausal women. JAMA 2002;288:2300-2306.

28. Moayyeri A, Besson H, Luben RN, Wareham NJ, Khaw KT. The association between physical activity in different domains of life and risk of osteoporotic fractures. Bone 2010;47:693-700.

29. Gregg EW, Cauley JA, Seeley DG, Ensrud KE, Bauer DC. Physical activity and osteoporotic fracture risk in older women. Study of Osteoporotic Fractures Research Group. Ann Intern Med 1998;129:81-88.

30. Englund U, Nordstrom P, Nilsson J, et al. Physical activity in middle-aged women and hip fracture risk: The UFO study. Osteoporos Int 2011;22:499-505.

31. Marks R. Physical activity and hip fracture disability: A review. J Aging Res 2011;2011:741918.

32. Lai JK, Lucas RM, Armstrong M, Banks E. Prospective observational study of physical functioning, physical activity, and time outdoors and the risk of hip fracture: A population-based cohort study of 158,057 older adults in the 45 and up study. J Bone Miner Res 2013;28:2222-2231.
33. Edwards MH, Gregson CL, Patel HP, et al. Muscle size, strength, and physical performance and their associations with bone structure in the Hertfordshire Cohort Study. J Bone Miner Res 2013;28:2295-2304.

34. Moayyeri A, Soltani A, Tabari NK, Sadatsafavi M, Hossein-Neghad A, Larijani B. Discordance in diagnosis of osteoporosis using spine and hip bone densitometry. BMC Endocr Disord 2005;5:3.

35. Ahmed LA, Center JR, Bjornerem A, et al. Progressively increasing fracture risk with advancing age after initial incident fragility fracture: The Tromso Study. J Bone Miner Res 2013;28:2214-2221.

36. Kruavit A, Chailurkit LO, Thakkinstian A, Sriphrapradang C, Rajatanavin R. Prevalence of vitamin D insufficiency and low bone mineral density in elderly Thai nursing home residents. BMC Geriatr 2012;12:49.

37. Harinarayan CV, Sachan A, Reddy PA, Satish KM, Prasad UV, Srivani P. Vitamin D status and bone mineral density in women of reproductive and postmenopausal age groups: a cross-sectional study from south India. J Assoc Physicians India 2011;59:698-704.

38. Morrow JR, Jr., Defina LF, Leonard D, Trudelle-Jackson E, Custodio MA. Meeting physical activity guidelines and musculoskeletal injury: The WIN study. Med Sci Sports Exerc 2012;44:1986-1992.

39. Kaplan RM, Herrmann AK, Morrison JT, Defina LF, Morrow J, Jr. Costs associated with women's physical activity musculoskeletal injuries: The women's injury study. J Phys Act Health 2014;11:1149-1155.

Address correspondence to: Laura F. DeFina, MD The Cooper Institute 12330 Preston Road Dallas, TX 75230

E-mail: ldefina@cooperinst.org 